\title{
Magnetic resonance imaging of an aortopulmonary window type three, with aortic atresia and interrupted aortic arch type B
}

\author{
Melanie Vogel, Dietmar Schranz, Juergen Bauer \\ Department of Paediatric Cardiology and Cardiac Surgery, Paediatric Heart Center, Justus-Liebig-University \\ Giessen, Germany
}

Received: 29 May 2011; Accepted: 23 August 2011; First published online: 21 September 2011

A 3700-GRAM GIRL BORN AT 39 WEEKS AND 4 days of gestation presented with a murmur on day 2 of life. The child appeared phenotypically normal with no dysmorphic features. An echocardiographic examination revealed a situs solitus with D-loop of the great vessels and aortic atresia with a large aortopulmonary window type three, and an interrupted left aortic arch type B. The descending aortic arch was interrupted below the left common carotid artery. A common feature of interrupted aortic arch is that the ascending aorta goes straight up cranially and does not begin to curve at the brachiocephalic vessel giving a " $\mathrm{Y}$ " or "two-pronged fork" appearance, with the aorta dividing into the first two brachiocephalic vessels that is, innominate or right common carotid and left common carotid - Figures 1 and 2. The magnetic resonance imaging demonstrates this sign and the complex anatomy clearly, with a three-dimensional reconstruction of this rare defect, which is important for surgical planning.

Shortly after diagnosis, a prostaglandin E1 infusion was commenced, as well as diuretics for preload reduction. On day 12 of life, bilateral pulmonary artery banding with a 4-millimetre polytetrafluoroethylene band was performed for reduction of pulmonary artery blood flow. At operation, the thymus was noted. The operation was uneventful and the electrocardiogram post surgery showed no signs

Correspondence to: Dr M. Vogel, Department of Paediatric Cardiology and Cardiac Surgery, Paediatric Heart Centre, Justus-Liebig University, Giessen, Feulgenstrasse 12, 35392 Giessen, Germany. Tel: 049641 9943461; Fax: 049 641 9943469; E-mail: mdrvogelv@aol.com of ischaemia. One day after surgery, the child developed a circulatory arrest and the post-mortem examination revealed fresh myocardial necrosis

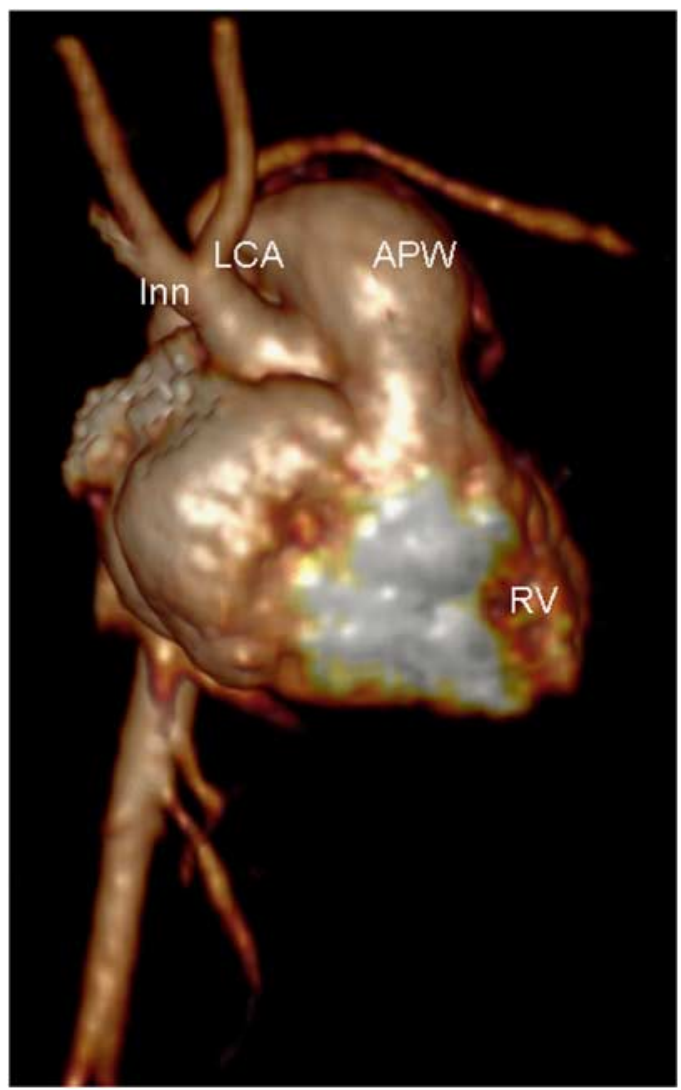

Figure 1.

$A P W=$ aortopulmonary window, Inn $=$ innominate artery, $L C A=$ left common carotid artery, $R V=$ right ventricle. 
Vol. 22, No. 2

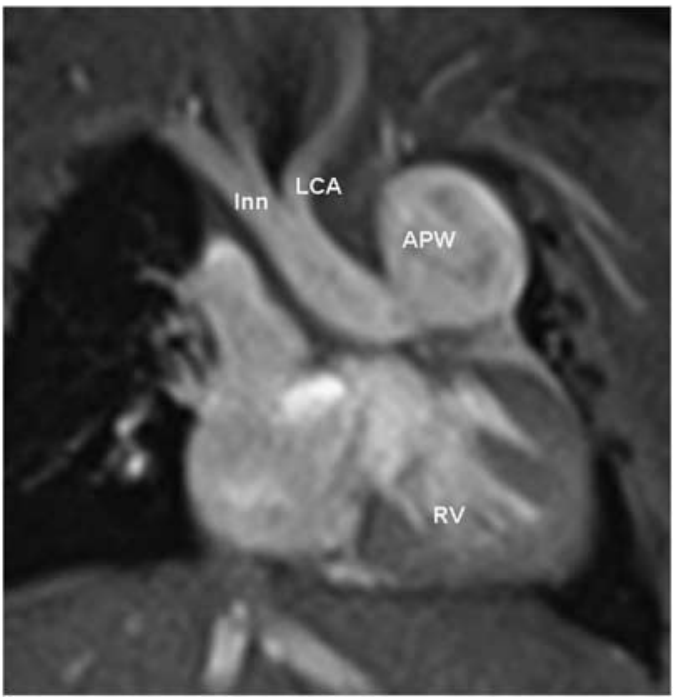

indicating a myocardial infarction. Despite the diagnostic advances of magnetic resonance imaging, this rare congenital heart defect carries a poor prognosis. ${ }^{1}$

\section{Reference}

1. Yew G, Coleman D, Calder L. Aortic valvar atresia, interrupted aortic arch, and a quadricuspid pulmonary valve: a rare combination. Pediatr Cardiol 2005; 26: 455-459.

Figure 2.

$A P W=$ aortopulmonary window, Inn $=$ innominate artery, $L C A=$ left common carotid artery, $R V=$ right ventricle. 